\title{
La conservación de la diversidad genética argentina: tres desafíos para implementar el régimen de acceso a los recursos genéticos y la distribución de los beneficios
}

\author{
LUCIANA C. SiLVESTRI \\ Cátedra UNESCO de Territorio y Medio Ambiente - Universidad Rey Juan Carlos, España
}

[Palabras clave: ABS, biodiversidad, Convenio sobre la Diversidad Biológica, Protocolo de Nagoya, biotecnología, biopiratería]

[Keywords: ABS, biodiversity, Convention on Biological Diversity, Nagoya Protocol, biotechnology, biopiracy]

\section{ACCESO A RECURSOS GENÉTICOS Y DISTRIBUCIÓN DE BENEFICIOS}

Las oportunidades que un régimen efectivo y eficiente de acceso a recursos genéticos y distribución de beneficios ("ABS", del inglés Access and Benefit-Sharing) podría lograr para un país proveedor de recursos genéticos como Argentina son varias. Aquí sólo destacaré tres en razón de brevedad y de su importancia. Por un lado, el mecanismo sería capaz de impulsar la conservación y utilización sostenible de la diversidad genética mediante la participación en los beneficios que surjan de la investigación y el desarrollo biotecnológico que se lleve a cabo sobre ella (Godt 2009). Por otra parte, si la mencionada distribución de beneficios privilegiara el acceso y transferencia de tecnología y la cooperación y colaboración en investigación, se promovería el avance del sector científico nacional y el achicamiento de la brecha tecnológica existente entre el norte y el sur (Barber et al. 2002). Por último, el ABS podría ser útil para recompensar a las comunidades indígenas y locales por el uso de su conocimiento tradicional en relación con la biodiversidad. Si bien esa información ha sido utilizada frecuentemente para el avance de la ciencia, estas comunidades nunca han sido retribuidas (Schuster 2001).

Sin embargo, la implementación nacional de un régimen de $A B S$ supone grandes dificultades que incluyen la escasa capacidad humana e institucional para conceder acceso y negociar contratos para el reparto de beneficios
(Lewis-Lettington et al. 2006), la existencia de vacíos legales (Chishakwe \& Young 2003) y el enquistamiento en posturas sólo enfocadas en beneficios monetarios en detrimento de otras oportunidades más realizables al corto plazo como por ejemplo, la colaboración científica (Glowka 2000).

Esta contribución, actual de cara a la próxima ratificación del Protocolo de Nagoya sobre acceso a los recursos genéticos y participación justa y equitativa en los beneficios que se deriven de su utilización por parte de la Argentina, pretende arrojar luz sobre los desafíos que enfrenta el país para implementar un régimen de acceso a recursos genéticos y distribución de beneficios. Luego de un breve repaso de la normativa internacional en la que se sustenta el ABS -útil para entender la estructura y funcionamiento del mecanismo- presentaré y analizaré los tres desafíos principales que a mi entender presenta el país en relación con el tema.

\section{ANCLAJE NORMATIVO INTERNACIONAL}

El ABS se encuentra regulado y desarrollado a nivel internacional en dos instrumentos principales: el Convenio sobre la Diversidad Biológica (CDB) y el Protocolo de Nagoya. El $\mathrm{CDB}$, que entró en vigor el 29 de diciembre de 1993, reconoce la soberanía de los Estados sobre los recursos naturales que se encuentran bajo su jurisdicción. En consecuencia, tienen la potestad para regular el acceso a sus recursos genéticos si lo consideran conveniente. Si un 
Estado decide reglamentarlo, quien desee acceder a los recursos deberá contar con su consentimiento fundamentado previo y acordar las condiciones del acceso y uso. Con respecto al conocimiento tradicional en posesión de comunidades indígenas y/o locales, el CDB establece la obligación de fomentar el reparto equitativo de los beneficios derivados de su utilización con las comunidades proveedoras de tal conocimiento.

Como se puede apreciar, si bien el CDB adelantó una serie de medidas apropiadas para los Estados proveedores de recursos genéticos, no alumbró nuevas y efectivas medidas de control del lado de los países usuarios. Con esto el problema estaba resuelto de forma parcial ya que no se exigía acreditar en el país del usuario de recursos genéticos y/o de conocimientos tradicionales que éstos hubieran sido accedidos legalmente (es decir, de acuerdo a la legislación nacional del país proveedor). Ese fue justamente el desafío que estaba llamado a solucionar el Protocolo de Nagoya, adoptado el 30 de octubre de 2010 en Nagoya, Japón.

El Protocolo, en reconocimiento de los derechos soberanos que los países ejercen sobre sus recursos naturales -incluidos los genéticos- busca que el acceso a ellos se produzca sólo cuando medie el consentimiento fundamentado previo del país proveedor y una distribución justa y equitativa de beneficios, concretada en términos mutuamente acordados, entre el solicitante y aquél. Entre los beneficios a acordar, que pueden ser monetarios y no monetarios, el Protocolo privilegia el acceso y transferencia de tecnología a favor del país proveedor y la colaboración y cooperación científica con él. Asimismo, el Protocolo de Nagoya regula el acceso al conocimiento tradicional asociado a la utilización de recursos genéticos en posesión de comunidades indígenas y/o locales, disponiendo que tal acceso se realice únicamente cuando medie consentimiento previo de las comunidades y con su participación en los beneficios que pudieran derivarse del uso del conocimiento. Hasta aquí el Protocolo repite, si bien con mayor detalle, lo ya establecido por el CDB.

Sin embargo, el Protocolo innova al obligar a los Estados a establecer "medidas de cumplimiento" que aseguren que los recursos genéticos y conocimientos tradicionales que sean utilizados dentro de su jurisdicción hayan sido accedidos de conformidad con la legislación nacional del país proveedor. También los obliga a adoptar medidas para abordar situaciones de incumplimiento con respecto a las medidas de cumplimiento anteriormente mencionadas.

El instrumento internacional individualiza una medida que siempre deberá adoptarse: la designación de un "punto de verificación" que controle la utilización de recursos genéticos en la propia jurisdicción. No especifica qué entidad deberá desempeñar este rol, pero el organismo que se designe deberá resultar pertinente a la utilización de recursos genéticos, o a la recopilación de información pertinente, en cualquier etapa de investigación, desarrollo, innovación, pre-comercialización o comercialización, pudiéndose facultar a la oficina nacional de patentes, el organismo nacional encargado de financiarinvestigación sobre recursosgenéticos u otro organismo relevante a la utilización de recursos genéticos. También es novedosa la introducción del "permiso", el documento que acredita el cumplimiento del consentimiento fundamentado previo y el establecimiento de condiciones mutuamente acordadas con el país proveedor. El permiso, que luego se convertirá en el "certificado internacional de cumplimiento", será exigible en la jurisdicción del usuario del recurso genético en los puntos de verificación establecidos.

\section{PRIMER DESAFÍO: El ESTADO ACTUAL DE LA LEGISLACIÓN}

El primer gran desafío que enfrenta el país para implementar un régimen jurídico sobre ABS que resulte estratégico, efectivo y que cumpla con las disposiciones del Protocolo de Nagoya, es, precisamente, el desarrollo limitado de la legislación nacional sobre el tema. En este sentido, se afirma que el marco legal (conformado por las Resoluciones $\mathrm{N}^{\circ}$ 226/2010 de la Secretaría de Ambiente y Desarrollo Sustentable que establece el régimen de acceso a recursos genéticos y $\mathrm{N}^{o}$ 208/2011 del Consejo Federal de Medio Ambiente [COFEMA]) no supone una verdadera norma de presupuestos mínimos en materia ambiental -en este caso sobre ABS-, tal y como debería existir de acuerdo a la Constitución Nacional. La Resolución $\mathrm{N}^{\mathrm{o}} 208 / 2011$ se limita a reafirmar el derecho de las Provincias y las comunidades locales 
sobre los recursos naturales y genéticos y a requerir a los organismos científicos y técnicos, públicos o privados, que obtengan la autorización provincial correspondiente cuando inicien proyectos o suscriban contratos que involucren el estudio, la explotación comercial, industrial o la utilización de recursos genéticos. Ella no reglamenta de forma pormenorizada los elementos esenciales de un régimen de $\mathrm{ABS}$. Es decir, no dispone sobre el consentimiento fundamentado previo, las condiciones mutuamente acordadas, el acceso con fines de investigación no comercial o los posibles beneficios a negociar.

Por otro lado, si bien la Resolución N ${ }^{\circ}$ 226/ 2010 sí regula con algún nivel de detalle los anteriores elementos, su alcance es limitado ya que sólo es aplicable cuando el propósito del acceso es la importación o exportación posterior de los recursos genéticos. Por lo tanto, la mayoría de los casos de acceso quedan fuera de su ámbito de aplicación; es decir, los que realizan las instituciones científicas nacionales con el fin de investigar los recursos genéticos del país en el país.

Además, el marco normativo nacional no regula el acceso al conocimiento tradicional asociado a la utilización de recursos genéticos en posesión de comunidades indígenas y/o locales. Sólo la Resolución N² 226/2010, en el marco de su limitado alcance, exige que el acuerdo entre partes presente "elementos que aseguren el respeto, preservación y mantenimiento de los conocimientos, innovaciones y prácticas de las comunidades indígenas y locales". Pero más allá de tal disposición no existe regulación adicional. Tal carencia, impensada en un régimen efectivo y estratégico sobre ABS, vulnera la Constitución Nacional, que reconoce la preexistencia étnica y cultural de los pueblos indígenas argentinos y garantiza - concurrentemente con las Provincias- el respeto a su identidad y su participación en la gestión referida a sus recursos naturales y a los demás intereses que los afecten.

En un mayor nivel de detalle, el marco normativo nacional presenta algunas ventajas y varias desventajas. Entre las primeras se puede incluir - siempre considerando el alcance limitado que tiene la Resolución $\mathrm{N}^{\circ} 226 / 2010$ - que ella prevé el cambio de condiciones posterior al momento en que se produce el acceso a recursos genéticos, estipulando que tal situación trae aparejada la necesidad de tramitar un nuevo permiso de acceso. Este bien podría ser el caso en que se autoriza una utilización meramente investigativa de recursos genéticos y luego existe intención de darles una finalidad comercial. Tal previsión se encuentra por otro lado de acuerdo con lo dispuesto por el Protocolo de Nagoya. Entre las desventajas, ninguna de las dos resoluciones incorpora en su ámbito material, posiblemente porque ambas son anteriores a la adopción del Protocolo de Nagoya, a los "derivados"; es decir, al compuesto bioquímico que existe naturalmente producido por la expresión genética o el metabolismo de los recursos biológicos o genéticos, incluso aunque no contenga unidades funcionales de la herencia. Los aromas usados por la industria cosmética y los bioquímicos útiles para el sector farmacéutico son algunos ejemplos. La inclusión de los "derivados" al régimen de ABS es central, pues el verdadero potencial económico de las bio-ciencias ha dejado de estar en la investigación del material genético en sí mismo para pasar al análisis de los compuestos bioquímicos que ocurren de manera natural como resultado de la expresión genética o el metabolismo de los recursos biológicos y genéticos.

El hecho de que ninguna de las dos resoluciones establezca oportunidades para el desarrollo del sector biotecnológico nacional constituye una debilidad. La legislación no privilegia el acceso y transferencia de tecnología y la colaboración y cooperación científica como beneficios a alcanzar en el marco de la distribución de beneficios. Tampoco requiere que las personas o instituciones de investigación extranjeras se hagan acompañar por una institución nacional de carácter científico para las actividades de acceso a recursos genéticos, requisito que podría servir para promover la comunidad científica del país. Así mismo, la falta de procedimientos simplificados y diferentes (expeditos) para el acceso a recursos genéticos con fines puramente científicos es una falencia grave del sistema legal porque burocratiza sobremanera la investigación con estas finalidades. En cualquier caso, tal diferenciación es ahora exigida por el Protocolo de Nagoya. Por último, el marco normativo argentino no presenta tres elementos esenciales introducidos por el Protocolo: 1) el "permiso" que certifica el cumplimiento del marco nacional del país 
proveedor en relación con el acceso a los recursos genéticos; 2) faltan "puntos de verificación" que controlen la legalidad del acceso a recursos genéticos provenientes de terceros países y que sean utilizados en nuestra jurisdicción; y 3) no existen "medidas de cumplimiento" para verificar que el acceso a recursos genéticos provenientes de otros países y al conocimiento tradicional a ellos asociado en posesión de comunidades indígenas y/o locales que residen en terceros países se haya realizado de conformidad con la norma extranjera.

\section{SEgundo DESAFíO: DesarRollo NORMATIVO A NIVEL PROVINCIAL}

El desarrollo legislativo a nivel provincial es escaso. Tan sólo ocho provincias de las 23 lo han regulado, lo que llama la atención pues a ellas les corresponde el dominio originario de sus recursos naturales de acuerdo a la ConstituciónNacional.Entonces, las provincias deberían tener el mayor interés en contar con una legislación efectiva y eficiente sobre ABS que les permitiera negociar contratos $-\mathrm{y}$ los consiguientes beneficios- para el acceso a sus recursos genéticos. La falta de regulación a nivel provincial es explicable en parte por la inexistencia a nivel nacional de una norma de presupuestos mínimos sobre ABS que permita profundización y desarrollo. Por otro lado, el desconocimiento sobre el tema en la mayoría de las provincias sea, posiblemente, otra de las causas que explican la ausencia de normas estratégicas.

Sólo Misiones, Jujuy, La Rioja, San Luis, La Pampa, Neuquén, Río Negro y Tierra del Fuego cuentan con legislación de ABS, si bien el grado de detalle entre unas y otras varía (Tabla 1). Por ejemplo, únicamente San Luis, Jujuy, Neuquén y Tierra del Fuego tienen una regulación pormenorizada, mientras que las demás sólo habilitan la regulación y / o sientan los criterios para la futura legislación. En este último sentido, la disposición más frecuente es la que expresa que los recursos genéticos pertenecen al dominio público de la Provincia, una aclaración vana pues sólo repite lo ya establecido por la Constitución Nacional.

Algunas de las provincias que regularon en detalle el ABS han impreso a sus legislaciones un marcado sesgo monetario. Tal es el caso de San Luis y Neuquén. Dicha priorización resulta inexplicable pues la experiencia demuestra que las expectativas de cuantiosos flujos de dinero fluyendo hacia los países proveedores de recursos genéticos a cambio del acceso a los mismos no se han cumplido (Correa 2005), y que por ello se deberían privilegiar beneficios más tangibles. Inclusive resulta curioso que la legislación neuquina establezca un porcentaje de pago de regalías tan alto como el $20 \%$ de la base imponible del Impuesto a los Ingresos Brutos para quien acceda a un recurso genético con fines comerciales o industriales (Ley $\mathrm{N}^{\mathrm{o}} 2.503$ ). Tal porcentaje desconoce que el verdadero valor agregado en biotecnología ocurre durante las etapas de investigación y desarrollo y no en la fase más simple de acceso a recursos genéticos. Por consiguiente, la voluntad de pago en dicho período inicial, donde todavía se desconoce cuáles serán los resultados de la investigación, es escasa. En cambio, Jujuy y Tierra del Fuego han logrado priorizar beneficios que promueven el desarrollo de las instituciones locales de investigación a través de la colaboración científica que debe darse entre los investigadores extranjeros que requieren acceso y sus pares locales. Dichos beneficios, más probables, promisorios y realizables en el corto plazo, parecen más realistas.

Por otra parte resulta ventajoso que algunas legislaciones provinciales hayan creado registros en relación con el ABS. Así por ejemplo, San Luis y Jujuy establecen un registro para el relevamiento de la diversidad genética provincial y otro en el que se deja constancia de las solicitudes de acceso y permisos acordados. Estos registros permiten conocer la verdadera demanda de recursos y tomar decisiones estratégicas en consecuencia.

\section{TERCER DESAFÍO: CAPACIDAD DE GESTIÓN Y VISIÓN ESTRATÉGICA}

La Argentina enfrenta una escasa capacidad de gestión para la implementación de un régimen de ABS. Si bien la Secretaría de Ambiente y Desarrollo Sustentable de la Nación posee suficiente conocimiento y experiencia, gracias -entre otras cosas- a los más de 4000 permisos de acceso acordados, varias circunstancias obstaculizan una gestión eficiente y estratégica. Entre ellas, la escasez de personal (sólo un funcionario está dedicado a recibir, evaluar y autorizar las solicitudes de acceso); La falta de concreción del registro de usuarios, solicitudes y permisos acordados; el 
limitado enlace y retroalimentación entre la Secretaría y la comunidad científica del país; la imposibilidad de acceso a bases de datos de derechos de propiedad intelectual, en trámite $\mathrm{o}$ acordados, nacionales o extranjeras, que pudiera servirle a la Secretaría para monitorear el patentamiento de productos e innovaciones basados en recursos genéticos argentinos; la falta de compilación, actualización y adecuación de códigos de conducta y mejores prácticas sobre ABS para los diferentes sectores de la investigación y producción; y la carencia de una página web completa y actualizada sobre ABS.

A nivel provincial, las capacidades de las administraciones para otorgar consentimiento fundamentado previo y negociar condiciones mutuamente son en general limitadas. En este contexto, se infiere una relación directa entre la mayor capacidad de las autoridades y la existencia efectiva de legislación provincial, como demuestran el caso de Jujuy y de Tierra del Fuego. Así mismo las capacidades provinciales para monitorear el acceso y utilización de recursos genéticos resultan insuficientes.

Finalmente, una razón subyacente a todos los desafíos expresados es la falta de visión estratégica sobre lo que el ABS podría lograr para el país. En este sentido, no percibo un posicionamiento claro e integral sobre las metas y objetivos que quisiéramos alcanzar en relación con la conservación y uso sustentable de nuestra diversidad genética $y$, a partir de allí, sobre cómo utilizar un mecanismo de incentivo como el ABS para conseguirlos. Igualmente, la escasa o nula sinergia que se observa en la planificación de las políticas o estrategias nacionales de diferentes sectores como por ejemplo, la de biodiversidad, ciencia y tecnología, asuntos indígenas y agricultura, permite afirmar que se pierden valiosísimas oportunidades para "matar dos pájaros de un tiro" mediante la utilización de un sistema tal como el de ABS. Por último, entiendo que la falta de prioridad que sufre la conservación de la diversidad genética del país en la agenda política termina por retrasar o paralizar decisiones importantes respecto a la implementación de un régimen de ABS a nivel nacional.

\section{CONCLUSIONES}

La Argentina debería reforzar tres ejes para la implementación de un régimen de ABS efectivo, estratégico y que satisfaga las obligaciones del Protocolo de Nagoya. El primero es adoptar un marco legislativo nacional, basado en el instrumento internacional, que siente presupuestos mínimos para el acceso a recursos genéticos y conocimiento tradicional asociado a su

Tabla 1. Síntesis de la legislación provincial sobre ABS en la República Argentina.

Table 1. Summary of provincial legislation on ABS in Argentine.

\begin{tabular}{|c|c|c|c|c|c|c|}
\hline Provincia & Norma & $\begin{array}{l}\text { Habilita } \\
\text { regulación del } \\
\text { ABS }\end{array}$ & $\begin{array}{l}\text { Requiere } \\
\text { consentimiento } \\
\text { fundamentado } \\
\text { previo }\end{array}$ & $\begin{array}{l}\text { Requiere } \\
\text { condiciones } \\
\text { mutuamente } \\
\text { acordadas }\end{array}$ & $\begin{array}{l}\text { Posee cláusulas } \\
\text { promueven sector } \\
\text { científico }\end{array}$ & $\begin{array}{l}\text { Crea registro } \\
\text { en relación con } \\
\text { el ABS }\end{array}$ \\
\hline Misiones & $\begin{array}{l}\text { Decreto } N^{\circ} \\
474 / 2002\end{array}$ & & $\begin{array}{l}\text { Sí } \\
\text { (art. 56) }\end{array}$ & $\begin{array}{l}\text { Sí } \\
\text { (art. 56) }\end{array}$ & $\begin{array}{l}\text { Sí } \\
\text { (arts. } 53 \text { y } 54 \text { ) }\end{array}$ & No \\
\hline Jujuy & $\begin{array}{l}\text { Res. } N^{\circ} 15 / 2003 \\
\text { Sec. Gestión } \\
\text { Ambiental }\end{array}$ & & $\begin{array}{l}\text { Sí } \\
\text { (art. 2) }\end{array}$ & $\begin{array}{l}\text { Sí } \\
\text { (art. 21) }\end{array}$ & $\begin{array}{l}\text { Sí } \\
\text { (arts. 2, } 19 \text { y } 20 \text { ) }\end{array}$ & $\begin{array}{l}\text { Sí } \\
\text { (art. 26) }\end{array}$ \\
\hline La Rioja & Ley N $\mathrm{N}^{\mathrm{o}} 7.801$. & $\begin{array}{l}\text { Sí } \\
\text { (art. 26) }\end{array}$ & No & No & No & No \\
\hline San Luis & $\begin{array}{l}\text { Ley No IX-0851- } \\
2013\end{array}$ & & $\begin{array}{l}\text { Sí } \\
\text { (art. 2) }\end{array}$ & $\begin{array}{l}\text { Sí } \\
\text { (art. 10) }\end{array}$ & $\begin{array}{l}\text { Sí } \\
\text { (art. 16) }\end{array}$ & $\begin{array}{l}\text { Sí } \\
\text { (arts. } 22 \text { y 23) }\end{array}$ \\
\hline La Pampa & Ley $\mathrm{N}^{\mathrm{o}} 1.914$ & $\begin{array}{l}\text { Sí } \\
\text { (art. 24c) }\end{array}$ & $\begin{array}{l}\text { Sí } \\
\text { (art. 24c) }\end{array}$ & No & No & No \\
\hline Neuquén & Ley $\mathrm{N}^{\mathrm{o}} 2.503$ & & $\begin{array}{l}\text { Sí } \\
\text { (art. 6) }\end{array}$ & $\begin{array}{l}\text { Sí } \\
\text { (art. 7) }\end{array}$ & No & $\begin{array}{l}\text { Sí } \\
\text { (art. 17) }\end{array}$ \\
\hline Río Negro & Ley $N^{\circ} 2600$ & $\begin{array}{l}\text { Sí } \\
\text { (art. 8) }\end{array}$ & $\begin{array}{l}\text { Sí } \\
\text { (arts. } 4 \text { y 7), pero } \\
\text { falta reglamentación }\end{array}$ & No & No & $\begin{array}{l}\text { Sí } \\
\text { (art. 6) }\end{array}$ \\
\hline $\begin{array}{l}\text { Tierra del } \\
\text { Fuego }\end{array}$ & $\begin{array}{l}\text { Res. N }{ }^{\circ} 570 / 12 \\
\text { Sec. Des. Sust. y } \\
\text { Amb. }\end{array}$ & & $\begin{array}{l}\text { Sí } \\
\text { (art. 1) }\end{array}$ & No & $\begin{array}{l}\text { Sí } \\
\text { (art. 2) }\end{array}$ & $\begin{array}{l}\text { Sí } \\
\text { (art. 3) }\end{array}$ \\
\hline
\end{tabular}


utilización. Este marco debería incorporar los siguientes elementos esenciales: a) incluir a los "derivados", ya que en ellos radica actualmente el verdadero potencial económico de la biotecnología; b) privilegiar beneficios relacionados con el acceso y transferencia de tecnología y con la colaboración científica a favor de las instituciones nacionales de investigación; c) asegurar que el acceso al conocimiento tradicional en posesión de comunidades indígenas y/o locales se efectúe únicamente cuando medie su consentimiento fundamentado previo y luego de establecer condiciones mutuamente acordadas con ellas; d) disponer de un procedimiento diferente y expedito para el acceso a recursos genéticos con fines puramente científicos; e) incorporar el "permiso"; f) designar "puntos de verificación" apropiados; $y$ h) prever "medidas de cumplimiento" para controlar la legalidad del acceso a recursos genéticos provenientes de otros países y al conocimiento tradicional a ellos asociado en posesión de comunidades indígenas y/o locales localizadas en otras jurisdicciones.

El segundo eje consiste en lograr que todas las provincias adopten legislación sobre ABS, la que, a su vez, debería respetar los presupuestos mínimos que la Nación establezca en el futuro. Para esto convendría aprender de la experiencia de las provincias que más han avanzado. Para las provincias que ya cuentan con normas de ABS se debería lograr su desarrollo estratégico, privilegiando beneficios no monetarios que promuevan el desarrollo del sector investigador argentino.

Finalmente, se deberían mejorar las capacidades de gestión de las organizaciones administrativas. Ello incluye:

- dotar y formar mayores recursos humanos, capacitar a los científicos que negocian acuerdos de acceso a recursos genéticos y a las comunidades indígenas y locales que otorgan acceso a su conocimiento tradicional;
- reforzar la interacción entre el investigador de recursos genéticos y la autoridad que los administra e implantar recursos técnicos como por ejemplo, un registro de usuarios y/o de acceso a recursos genéticos a nivel nacional;

- garantizar el acceso a bases de datos de patentes nacionales y extranjeras; $y$

- mantener actualizada la página web de la Secretaría de Ambiente y Desarrollo Sustentable de la Nación.

Estas mejoras deberían darse en el marco del planteamiento y respuesta de una premisa esencial: ¿qué es lo queremos lograr como país con nuestros recursos genéticos y cómo puede ayudarnos para ello el régimen de ABS?

\section{BIBLIOGRAFÍA}

Barber, CV; L GlowKa \& A La Viña. 2002. Developing and implementing national measures for genetic resources access regulation and benefit-sharing. [12]. Pp. 363-414 en: LAIRD, SA (ed.). Biodiversity and Traditional Knowledge. Equitable Partnerships in Practice. Earthscan. London and Sterling, VA. Pp. 504.

Chishakwe, N \& TR Young. 2003. Access to Genetic Resources, and Sharing the Benefits of their Use: International and Subregional Issues. Informe inédito. IUCN. Pp. 18.

Correa, C. 2005. Do national access regimes promote the use of genetic resources and benefit sharing? Int. J. Environment and Sustainable Development, 4(4):444-463.

GLOWKA, L. 2000. Bioprospecting, Alien Invasive Species, and Hydrothermal Vents: Three Emerging Legal Issues in the Conservation and Sustainable Use of Biodiversity. Tulane Environmental Law Journal, 13(2):329-360.

GodT, C. 2009. Enforcement of Benefit- Sharing Duties in User Countries. [22]. Pp. 419-438 en: KamaU, EC \& G WINTER (eds.). Genetic Resources, Traditional Knowledge $\mathcal{E}$ The Law. Solutions for Access and Benefit Sharing. Earthscan. London and Sterling, VA. Pp. 494.

Lewis-Lettington, RJ; M Ruiz Muller; TR Young; KA Nnadozie; M Halewood, J Cabrera Medaglia. 2006. Methodology for Developing Policies and Laws for Access to Genetic Resources and Benefit Sharing. International Plant Genetic Resources Institute. Rome, Italy. Pp. 35.

SChuster, B. 2001. A New Integrated Program for Natural Product Development and the Value of an Ethnomedical Approach. The Journal of Alternative and Complementary Medicine, 7(6):61-72. 